\title{
Besondere Punkte der Euler-Geraden
}

\author{
OSWALD GIERING
}

\begin{abstract}
In the following article the concepts „Euler line of a triangle“ and „radical centre of three circles" are connected. In this way we could find some relations between special points of a triangle (orthocentre $H$, centre of gravity $S$, circumcentre $M$, midpoint $F$ of the nine-point circle) and the radical centres of special triples of circles.
\end{abstract}

Key words and phrases: geometry of triangles, remarkable points of a triangle, radical centre of three circles.

ZDM Subject Classification: G40.

\section{Einleitung}

Die Beiträge der Schulgeometrie zur Elementargeometrie nehmen seit Jahrhunderten einen breiten Raum ein. Zusammenfassende Darstellungen findet man in [5] und [6]. Der vorliegende Beitrag bedient sich einer bewährten Methode, um im Rahmen der Elementargeometrie Neues zu finden. Die Methode besteht darin, Begriffe (Fragestellungen, Ergebnisse) miteinander zu koppeln, die bisher unverknüpft nebeneinanderstehen. Als Beispiel dafür koppeln wir - kurz gesagt den Begriff Euler-Gerade eines Dreiecks (den wir in Abschnitt 2 vorstellen) mit dem Begriff Potenzpunkt von drei Kreisen (den wir in Abschnitt 3 vorstellen).

In den folgenden Abschnitten betrachten wir aus einem Dreieck $A B C$ abgeleitete Kreistripel, deren Potenzpunkte entweder auf der Euler-Geraden des Dreiecks $A B C$ liegen oder in anderer Weise mit Punkten der Euler-Geraden verknüpft sind (speziell mit dem Höhenschnittpunkt, dem Schwerpunkt, dem Umkreismittelpunkt und dem Mittelpunkt des Feuerbach-Kreises des Dreiecks $A B C$ ). 
Die Konfiguration dieser Potenzpunkte zeigt zahlreiche Kollinearitäten, Parallelitäten und Symmetrien, die in Abbildung 6 dargestellt sind. Wir arbeiten in der euklidischen Ebene, die bei Bedarf projektiv oder auch komplex erweitert wird.

\section{Euler-Gerade eines Dreiecks}

Über den Höhenschnittpunkt $H$, den Schwerpunkt $S$, den Umkreismittelpunkt $M$ und den Mittelpunkt $F$ des Feuerbach-Kreises eines Dreiecks $A B C$ gilt der folgende Satz ([3], S. 148ff):

SATZ 1 (L. Euler 1707-1783, K. W. Feuerbach 1800-1834). Ist das Dreieck $A B C$ nicht gleichseitig, dann liegen die Punkte $H, S, M, F$ auf einer Geraden (der Euler-Geraden des Dreiecks $A B C$ ) und es bestehen die Streckengleichheiten: $\overline{H S}=2 \overline{M S}, \overline{H M}=2 \overline{F M}, \overline{H F}=3 \overline{S F}, \overline{M S}=2 \overline{F S}$ (Abb. 1). Ist das Dreieck $A B C$ gleichseitig, so gilt $H=S=M=F$.

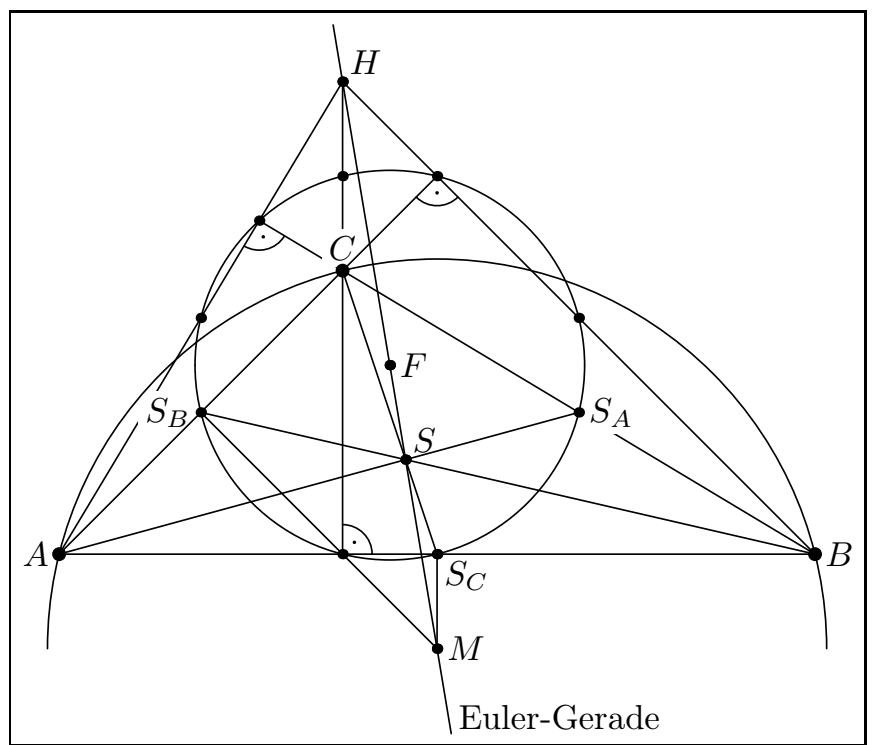

Abbildung 1

Die Punkte der Euler-Geraden heißen im Folgenden Euler-Punkte. 


\section{Potenzpunkt von drei Kreisen}

Zwei Kreisen $k_{1}, k_{2}$ mit verschiedenen Mittelpunkten $M_{1}, M_{2}$ läßt sich die Verbindungsgerade ihrer beiden eigentlichen (reell verschiedenen, reell zusammenfallenden oder konjugiert komplexen) Schnittpunkte zuordnen. Diese stets reelle Verbindungsgerade $p_{12}$ heißt die Potenzgerade (Chordale oder Radikalachse) der beiden Kreise ([3], S. 140, [4], S. 177). Sie ist orthogonal zur Verbindungsgerade der Kreismittelpunkte $M_{1}, M_{2}$ (Abb. 2). Die Potenzgerade konzentrischer Kreise ist die Ferngerade der Ebene ([4], S. 177).

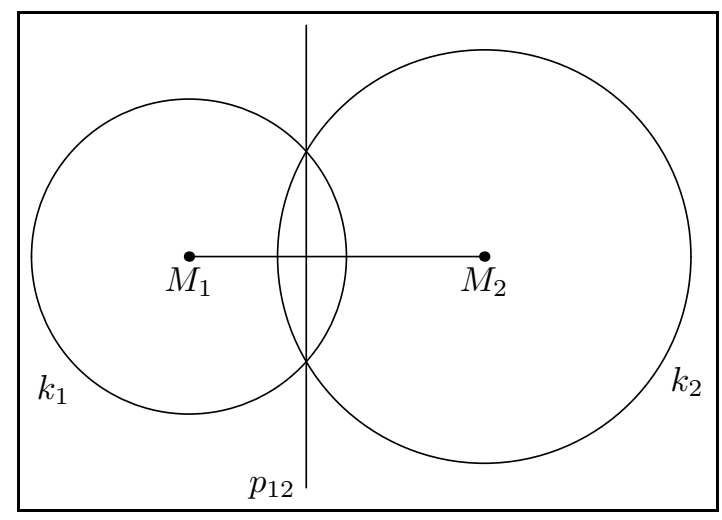

Abbildung 2

Die Potenzgerade zweier Kreise $k_{1}, k_{2}$ mit konjugiert komplexen eigentlichen Schnittpunkten läßt sich nach Abbildung 3 reell konstruieren: Man wähle einen Hilfskreis $k_{3}$, der sowohl $k_{1}$ als auch $k_{2}$ reell in zwei verschiedenen Punkten schneidet. Der Schnittpunkt der Potenzgeraden $p_{23}$ und $p_{31}$ ist dann ein Punkt der gesuchten (zu $M_{1} M_{2}$ orthogonalen) Potenzgeraden $p_{12}$. Diese Konstruktion verwendet bereits den folgenden Satz über den Potenzpunkt von drei Kreisen ([3], S. 140):

SAtz 2 (G. Monge 1746-1818). Seien $k_{1}, k_{2}, k_{3}$ Kreise mit paarweise verschiedenen Mittelpunkten $M_{1}, M_{2}, M_{3}$. Dann gilt: Sind $M_{1}, M_{2}, M_{3}$ nicht kollinear, dann sind die Potenzgeraden $p_{12}, p_{23}, p_{31}$ kopunktal (im Potenzpunkt $P$ der drei Kreise). Sind $M_{1}, M_{2}, M_{3}$ kollinear, dann sind die Potenzgeraden $p_{12}, p_{23}, p_{31}$ parallel (in ihrem Fernpunkt kopunktal). 


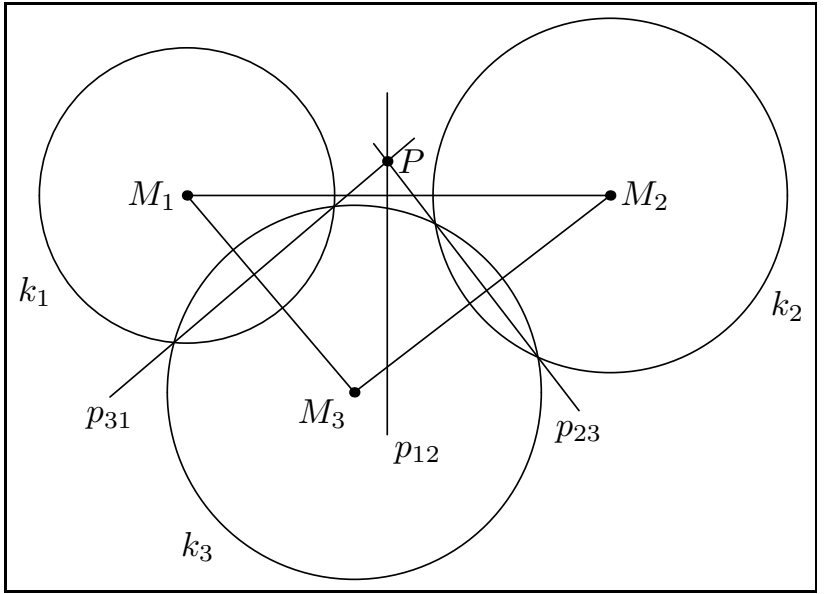

Abbildung 3

\section{Potenzpunkte auf der Euler-Geraden}

Nach Satz 2 wird je drei Kreisen $k_{1}, k_{2}, k_{2}$, deren Mittelpunkte ein Dreieck bilden, ihr (eigentlicher) Potenzpunkt $P$ zugeordnet (Abb. 3). Diese Zuordnung definiert eine Abbildung der $\infty^{9}$ (d.h. von 9 Parametern abhängigen ${ }^{1}$ ) Kreistripel $\left(k_{1}, k_{2}, k_{3}\right)$ auf die $\infty^{2}$ Punkte $P$ der Ebene. Es gibt somit „viele“ Kreistripel, die auf denselben Punkt abgebildet werden.

Wir betrachten nun solche Kreistripel, die sich aus einem gegebenen Dreieck $A B C$ ableiten lassen und deren Potenzpunkte auf der Euler-Geraden des Dreiecks $A B C$ liegen oder deren Potenzpunkte auf Punkte der Euler-Geraden führen. Da "viele" Kreistripel denselben Potenzpunkt besitzen, wird man Beschränkungen einführen, die interessante Kreistripel aussondern. Als Beispiel dafür betrachten wir im Folgenden jene Kreistripel, die sich

(A) allein mit Hilfe der Ecken $A, B, C$ des Dreiecks und seinen Seitenmitten $S_{A} \in \overline{B C}, S_{B} \in \overline{C A}, S_{C} \in \overline{A B}$ definieren lassen und die

(B) bei den zyklischen Vertauschungen $A \rightarrow B \rightarrow C \rightarrow A$ und $S_{A} \rightarrow S_{B} \rightarrow$ $S_{C} \rightarrow S_{A}$ in sich übergehen.

${ }^{1}$ Sechs der 9 Parameter dienen der Festlegung der drei Kreismittelpunkte, drei Parameter legen die Längen der Kreisradien fest. 
Die Dreiecke $A B C$ und $S_{A} S_{B} S_{C}$ besitzen denselben Schwerpunkt $S$ und dieselbe Euler-Gerade.

\section{Analytische Behandlung}

Zur analytischen Behandlung beziehen wir das Dreieck $A B C$ auf das in Abbildung 4 eingetragene kartesische $x y$-Koordinatensystem und geben den Ecken die Koordinaten $A(a, 0), B(b, 0), C(0, c)$. Ohne Einschränkung sei $a<b, 0<c$. Die Seitenmitten $S_{A}, S_{B}, S_{C}$ besitzen dann die Koordinaten:

$$
S_{A}\left(\frac{b}{2}, \frac{c}{2}\right), \quad S_{B}\left(\frac{a}{2}, \frac{c}{2}\right), \quad S_{C}\left(\frac{a+b}{2}, 0\right) .
$$

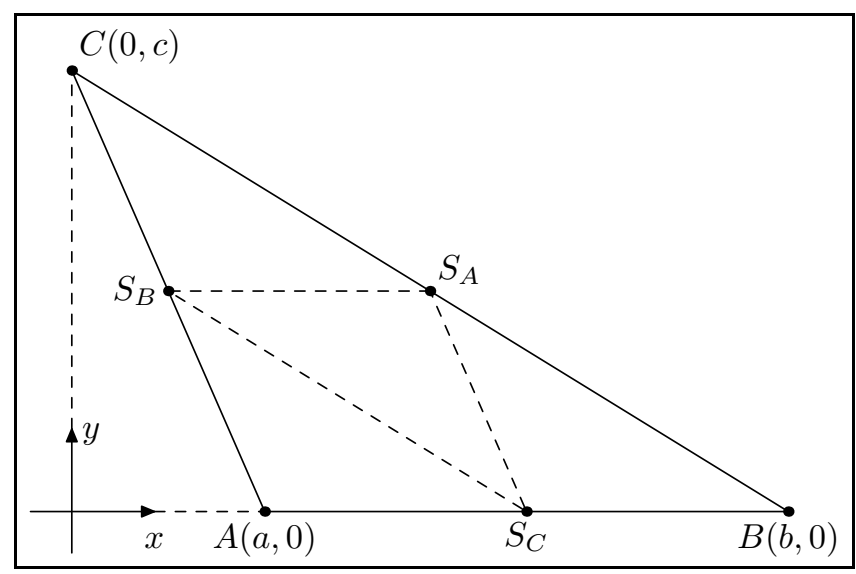

Abbildung 4

Der Kreis $k_{i}(i=1,2,3)$ mit Mittelpunkt $M_{i}\left(x_{i}, y_{i}\right)$ und Radius $r_{i}$ besitzt die Gleichung

$$
\left(x-x_{i}\right)^{2}+\left(y-y_{i}\right)^{2}=r_{i}^{2} .
$$

Die Gleichung der Potenzgeraden $p_{i j}$ der Kreise $k_{i}, k_{j}(i, j=1,2,3 ; i \neq j)$ lautet

$$
2\left(x_{j}-x_{i}\right) x+2\left(y_{j}-y_{i}\right) y+\left(r_{j}^{2}-x_{j}^{2}-y_{j}^{2}\right)-\left(r_{i}^{2}-x_{i}^{2}-y_{i}^{2}\right)=0 .
$$


Sind die drei Potenzgeraden $p_{12}, p_{23}, p_{31}$ der Kreise $k_{i}$ kopunktal im Potenzpunkt $P\left(x_{P}, y_{P}\right)$, dann berechnet man aus (2)

$$
\begin{aligned}
& x_{P}=\frac{1}{D}\left|\begin{array}{ll}
\left(y_{2}-y_{1}\right) & \left(x_{1}^{2}+y_{1}^{2}-r_{1}^{2}\right)-\left(x_{2}^{2}+y_{2}^{2}-r_{2}^{2}\right) \\
\left(y_{3}-y_{2}\right) & \left(x_{2}^{2}+y_{2}^{2}-r_{2}^{2}\right)-\left(x_{3}^{2}+y_{3}^{2}-r_{3}^{2}\right)
\end{array}\right| \\
& y_{P}=\frac{1}{D}\left|\begin{array}{ll}
\left(x_{1}^{2}+y_{1}^{2}-r_{1}^{2}\right)-\left(x_{2}^{2}+y_{2}^{2}-r_{2}^{2}\right) & \left(x_{2}-x_{1}\right) \\
\left(x_{2}^{2}+y_{2}^{2}-r_{2}^{2}\right)-\left(x_{3}^{2}+y_{3}^{2}-r_{3}^{2}\right) & \left(x_{3}-x_{2}\right)
\end{array}\right|
\end{aligned}
$$

mit $D=2\left[\left(x_{2}-x_{1}\right)\left(y_{3}-y_{2}\right)-\left(x_{3}-x_{2}\right)\left(y_{2}-y_{1}\right)\right]$. Als Gleichung der Euler-Geraden des Dreiecks $A B C$ findet man

$$
\left(c^{2}+3 a b\right) x-c(a+b) y=a b(a+b)
$$

Die auf der Euler-Geraden liegenden Punkte $H, S, M, F$ besitzen die Koordinaten

$$
H\left(0,-\frac{a b}{c}\right), S\left(\frac{a+b}{3}, \frac{c}{3}\right), M\left(\frac{a+b}{2}, \frac{c^{2}+a b}{2 c}\right), F\left(\frac{a+b}{4}, \frac{c^{2}-a b}{4 c}\right) .
$$

\section{Klassen von Kreistripeln}

Nun seien $K, K^{*}, K^{* *}$ drei Klassen von Kreistripeln, die den Bedingungen (A) und (B) genügen. Die Tripel der Klassen $K, K^{*}, K^{* *}$ seien der Reihe nach gebildet aus Kreisen folgenden Typs:

$$
\begin{array}{ll}
k(P, Q) & \text { Kreis mit Mittelpunkt } P, \text { Kreis durch den Punkt } Q, \\
k^{*}(\overline{P Q}) & \text { Kreis über der Strecke } \overline{P Q}, \\
k^{* *}(P, Q, R) & \text { Kreis durch die Punkte } P, Q, R .
\end{array}
$$

Die Punkte $P, Q, R$ werden nach (A) der Punktmenge $\left\{A, B, C, S_{A}, S_{B}, S_{C}\right\}$ entnommen. Sodann werden die zyklischen Vertauschungen (B) angewendet. Man findet

9 verschiedene Kreistripel in $K$, ihre Potenzpunkte seien $1,2, \ldots, 9$;

5 verschiedene Kreistripel in $K^{*}$, ihre Potenzpunkte seien $1^{*}, 2^{*}, \ldots, 5^{*}$;

5 verschiedene Kreistripel in $K^{* *}$, ihre Potenzpunkte seien $1^{* *}, 2^{* *}, \ldots, 5^{* *}$. 


\section{Die Potenzpunkte der Kreistripelklasse $K$}

Wir betrachten zunächst die Potenzpunkte 1,2, ., 9 der Kreistripel der Klasse $K$. In der folgenden Tabelle bezeichnet in jeder Zeile der links eingetragene Punkt den Potenzpunkt des rechts vermerkten Kreistripels. Dabei symbolisieren die Pfeile - wie auch in den folgenden Tabellen - die zyklische Vertauschung nach (B) der die Kreise definierenden Punkte.

\begin{tabular}{c|c} 
Potenzpunkt & Kreistripel \\
\hline 1 & $k(B, A) \rightarrow k(C, B) \rightarrow k(A, C)$ \\
2 & $k(A, B) \rightarrow k(B, C) \rightarrow k(C, A)$ \\
\hline 3 & $k\left(S_{A}, S_{B}\right) \rightarrow k\left(S_{B}, S_{C}\right) \rightarrow k\left(S_{C}, S_{A}\right)$ \\
4 & $k\left(S_{B}, S_{A}\right) \rightarrow k\left(S_{C}, S_{B}\right) \rightarrow k\left(S_{A}, S_{C}\right)$ \\
\hline 5 & $k\left(A, S_{B}\right) \rightarrow k\left(B, S_{C}\right) \rightarrow k\left(C, S_{A}\right)$ \\
7 & $k\left(S_{B}, A\right) \rightarrow k\left(S_{C}, B\right) \rightarrow k\left(S_{A}, C\right)$ \\
\hline 6 & $k\left(A, S_{C}\right) \rightarrow k\left(B, S_{A}\right) \rightarrow k\left(C, S_{B}\right)$ \\
7 & $k\left(S_{C}, A\right) \rightarrow k\left(S_{A}, B\right) \rightarrow k\left(S_{B}, C\right)$ \\
\hline 8 & $k\left(A, S_{A}\right) \rightarrow k\left(B, S_{B}\right) \rightarrow k\left(C, S_{C}\right)$ \\
9 & $k\left(S_{A}, A\right) \rightarrow k\left(S_{B}, B\right) \rightarrow k\left(S_{C}, C\right)$
\end{tabular}

Tabelle (7) ist in aufeinanderfolgenden Zeilenpaaren notiert. In jedem Zeilenpaar sind die untereinander stehenden Kreise kongruent. Jedes Kreistripel mit dem Potenzpunkt 7 besteht aus den Kreisen über den Seiten des Dreiecks $A B C$. Nach Satz 2 lassen sich die Potenzpunkte 1,2, ., 9 in der Ebene des Dreiecks $A B C$ konstruieren. Abbildung 5 zeigt die Konstruktion des Potenzpunkts 2 der Kreistripel $k(A, B) \rightarrow k(B, C) \rightarrow k(C, A)$.

Berechnet man die Koordinaten der Kreismittelpunkte sowie die Radiusquadrate der Kreise in Tabelle (7), dann findet man nach (4) die Koordinaten der Potenzpunkte $1,2, \ldots, 9$. Als Koordinaten der Punkte 1, 3, 5 erhält man:

$$
\begin{aligned}
& 1\left(\frac{2 a b-a^{2}+c^{2}}{2(b-a)}, \frac{a^{2}(2 b-a)+b\left(a b-b^{2}+c^{2}\right)}{2 c(b-a)}\right) \\
& 3\left(\frac{2 a b-2 a^{2}+b^{2}+c^{2}}{4(b-a)}, \frac{a b(a+2 b)-a^{3}-b^{3}+(2 b-a) c^{2}}{4 c(b-a)}\right) \\
& 5\left(\frac{2 a b-4 a^{2}+3 b^{2}+c^{2}}{8(b-a)}, \frac{b^{2}(4 a-b)-a^{2}(a+b)-c^{2}(3 a-4 b)}{8 c(b-a)}\right)
\end{aligned}
$$




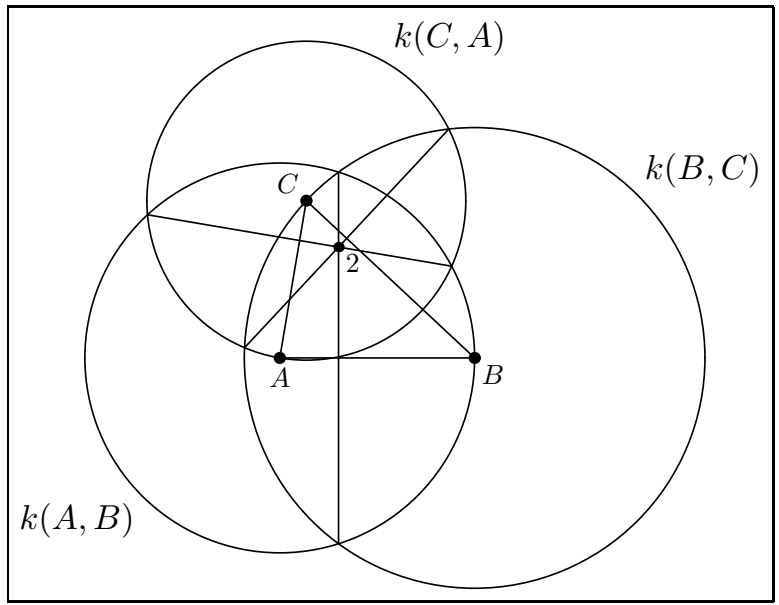

Abbildung 5

Die Koordinaten der Punkte 2, 4, 6 entstehen der Reihe nach aus den Koordinaten der Punkte $1,3,5$, indem man $a$ mit $b$ vertauscht und $c$ beibehält. ${ }^{2}$ Die Potenzpunkte 7, 8, 9 besitzen die Koordinaten:

$$
7\left(0,-\frac{a b}{c}\right), \quad 8\left(\frac{a+b}{8}, \frac{c^{2}-5 a b}{8 c}\right), \quad 9\left(a+b, \frac{c^{2}+2 a b}{c}\right)
$$

Wir formulieren nun in Satz 3 Aussagen über die Lage der Potenzpunkte 1, 2, ., 9 zu den Euler-Punkten $H, S, M, F$ :

SATz 3. Im nicht gleichseitigen Dreieck $A B C$ liegen die Potenzpunkte $1,2, \ldots, 9$ der Kreistripel (7) zu den Euler-Punkten $H, S, M, F$ wie folgt (Abb. 6):

(1.1) Der Mittelpunkt der Strecke $\overline{12}$ ist der Mittelpunkt $F$ des FeuerbachKreises und zugleich der Mittelpunkt der Strecke $\overline{H M}$.

(1.2) Der Mittelpunkt $Y$ der Strecke $\overline{34}$ ist der Mittelpunkt der Strecke $\overline{M F}$.

(1.3) Der Mittelpunkt $X$ der Strecke $\overline{56}$ ist der Mittelpunkt der Strecke $\overline{M Y}$.

(1.4) Der Potenzpunkt 7 ist der Höhenschnittpunkt $H$.

(1.5) Der Potenzpunkt 8 ist der Mittelpunkt der Strecke $\overline{H F}$.

(1.6) Der Potenzpunkt 9 ist der Spiegelpunkt von $H$ an $M$.

${ }^{2}$ Man erkennt dies durch Rechnung, aber auch aufgrund der Wahl des $x y$-Koordinatensystems und der die Potenzpunkte $1,2, \ldots, 6$ definierenden Kreistripel. 


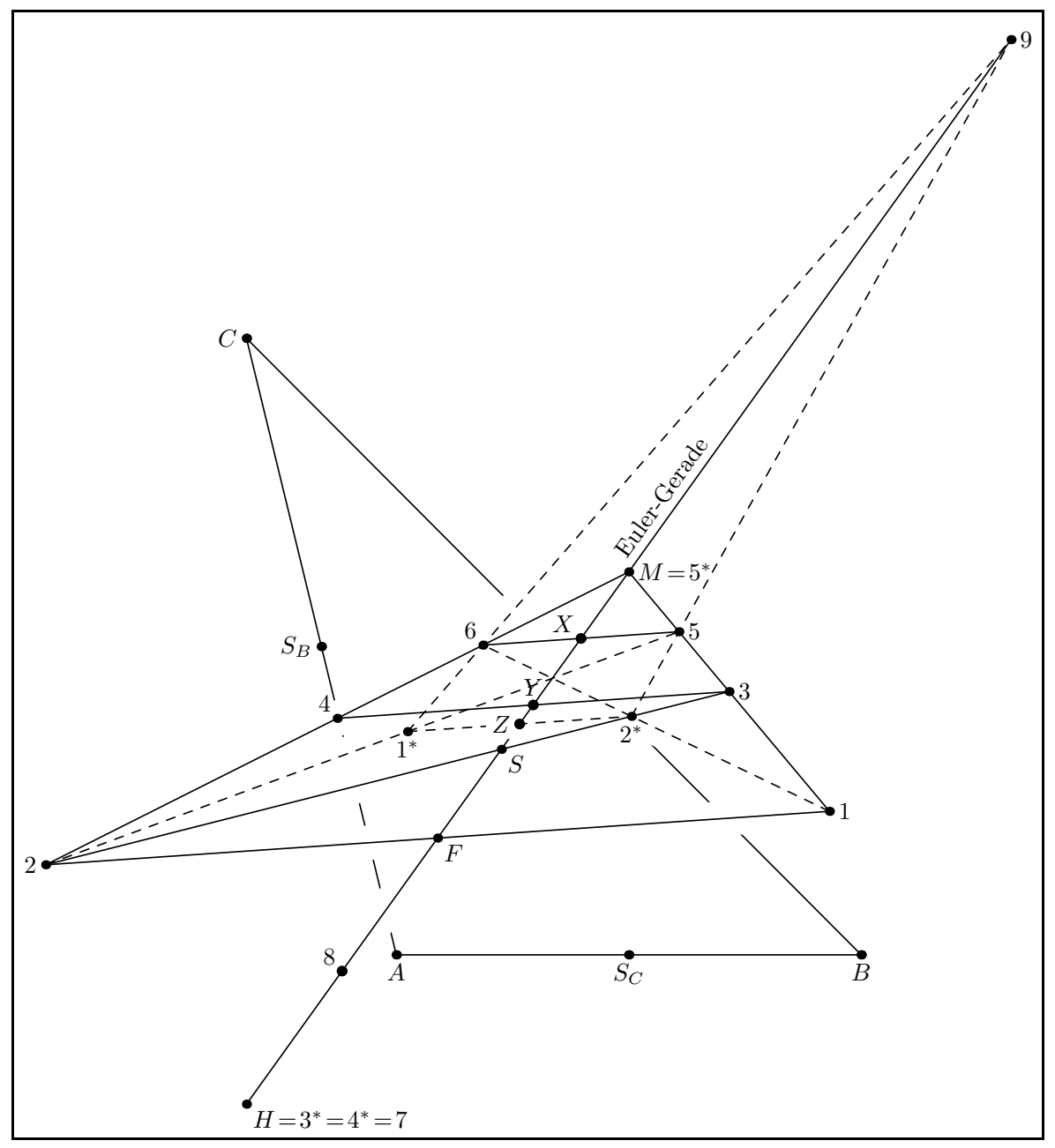

Abbildung 6

Die Mittelpunkte der Strecken $\overline{12}, \overline{34}, \overline{56}$ und die Potenzpunkte 7, 8, 9 sind folglich Euler-Punkte. Außerdem gilt:

(1.7) Die Punkte $M, 1,3,5$ sowie $M, 2,4,6$ sind kollinear.

(1.8) Die Punkte $S, 2,3$ sowie $S, 1,4$ sind kollinear.

(1.9) Die folgenden Geraden sind parallel: $12\|34\| 56$; $H 1\|M 2, M 1\| H 2 ; 63 \| 41$, $54\|32 ; M 3\| F 4, M 4\|F 3 ; H 4\| 91, H 3\|92 ; X 4\| 81, X 3 \| 82$. 
Im gleichseitigen Dreieck $A B C$ entarten (1.1)-(1.9) in leicht durchschaubarer Weise.

Die in Satz 3 enthaltenen Aussagen lassen sich - ausgehend von den Koordinatendarstellungen der Euler-Punkte $H, S, M, F$ und der Potenzpunkte 1,2, ., 9 - auf bekannte Art verifizieren. Aufgrund der Wahl des $x y$-Koordinatensystems sind die Koordinaten der Euler-Punkte $H, S, M, F, 7,8,9, X, Y$ invariant gegen Vertauschung von $a$ und $b$. Ausgehend von Satz 3 lassen sich weitere Aussagen rein elementargeometrisch gewinnen. So folgt zum Beispiel: Das Viereck H1M2 ist ein Parallelogramm (man verwende $\overline{H F}=\overline{F M}$ und (1.1) aus Satz 3). Die Dreiecke H34 und 129 sind ähnlich mit dem Ähnlichkeitszentrum $S$ (man verwende (1.8) und (1.9) aus Satz 3). S ist der gemeinsame Schwerpunkt der Dreiecke $A B C$ und M12 (man verwende (1.1), (1.2) und (1.9) aus Satz 3). Die Punkte M, 1, 3, 5 entstehen aus den Punkten $M, 2,4,6$ durch eine schiefe Spiegelung an der Euler-Geraden (man verwende (1.1), (1.2), (1.3) und (1.9) aus Satz 3).

\section{Die Potenzpunkte der Kreistripelklasse $K^{*}$}

Die Verknüpfung der Begriffe Euler-Gerade eines Dreiecks und Potenzpunkt von drei Kreisen führte in Abschnitt $7 \mathrm{zu}$ einer ersten Erweiterung der Konfiguration der Euler-Punkte $H, S, M, F$ um die Potenzpunkte 1,2, ., 9 mit den in Satz 3 genannten Eigenschaften. Wir betrachten nun eine zweite Erweiterung um die Potenzpunkte $1^{*}, 2^{*}, \ldots, 5^{*}$ der Kreistripelklasse $K^{*}$. Der Tabelle (7) entspricht nun die Tabelle:

\begin{tabular}{c|l} 
Potenzpunkt & \multicolumn{1}{|c}{ Kreistripel } \\
\hline $1^{*}$ & $k\left(\overline{A S_{C}}\right) \rightarrow k\left(\overline{B S_{A}}\right) \rightarrow k\left(\overline{C S_{B}}\right)$ \\
$2^{*}$ & $k\left(\overline{B S_{C}}\right) \rightarrow k\left(\overline{C S_{A}}\right) \rightarrow k\left(\overline{A S_{B}}\right)$ \\
\hline $3^{*}$ & $k(\overline{A B}) \rightarrow k(\overline{B C}) \rightarrow k(\overline{C A})$ \\
$4^{*}$ & $k\left(\overline{A S_{A}}\right) \rightarrow k\left(\overline{B S_{B}}\right) \rightarrow k\left(\overline{C S_{C}}\right)$ \\
$5^{*}$ & $k\left(\overline{S_{A} S_{B}}\right) \rightarrow k\left(\overline{S_{B} S_{C}}\right) \rightarrow k\left(\overline{S_{C} S_{A}}\right)$
\end{tabular}

In (10) sind die untereinander stehenden Kreise der ersten beiden Zeilen kongruent. Die 3. Zeile enthält die Kreise über den Seiten des Dreiecks $A B C$, die auch in (7) auftreten und den Potenzpunkt 7 besitzen. Die 4. Zeile enthält die Kreise über den Seitenhalbierenden des Dreiecks $A B C$, und in der 5. Zeile stehen die 
Kreise über den Seiten des Seitenmittendreiecks $S_{A} S_{B} S_{C}$. Als Koordinaten des Potenzpunkts $1^{*}$ berechnet man

$$
1^{*}\left(\frac{3 b^{2}-2 a^{2}-2 a b-c^{2}}{7(b-a)}, \frac{a^{3}+b^{3}-2 a^{2} b-a b^{2}+(2 b-3 a) c^{2}}{7 c(b-a)}\right)
$$

Die Koordinaten von $2^{*}$ entstehen aus den Koordinaten von $1^{*}$, indem man $a$ mit $b$ vertauscht und $c$ beibehält.

Satz 3 läßt sich nun ergänzen durch Satz 4:

SATz 4. Im nicht gleichseitigen Dreieck $A B C$ liegen die Potenzpunkte $1^{*}, 2^{*}, \ldots, 5^{*}$ der Kreistripel (10) zu den Euler-Punkten $H, S, M, F$ und den Potenzpunkten 1,2, ., 9 der Kreistripel (7) wie folgt (Abb. 6):

(2.1) Der Mittelpunkt $Z$ der Strecke $\overline{1^{*} 2^{*}}$ ist ein Euler-Punkt.

(2.2) Die Potenzpunkte $3^{*}$ und $4^{*}$ sind der Höhenschnittpunkt $H$.

(2.3) Der Potenzpunkt $5^{*}$ ist der Umkreismittelpunkt $M$.

Die Potenzpunkte $3^{*}, 4^{*}, 5^{*}$ sind folglich Euler-Punkte. Außerdem gilt:

(2.4) Die Punkte $S, 1,4,1^{*}$ sowie $S, 2,3,2^{*}$ sind kollinear.

(2.5) Die Punkte 6, 1, 2* sowie 5, 2, $1^{*}$ sind kollinear.

(2.6) Die Punkte 5, 9, 2* sowie 6, 9, $1^{*}$ sind kollinear.

(2.7) Die folgenden Geraden sind parallel: $1^{*} 2^{*}\left\|34, M 1^{*}\right\| 38, M 2^{*} \| 48$.

Im gleichseitigen Dreieck $A B C$ entarten (2.1)-(2.7) in leicht durchschaubarer Weise.

In der Konfiguration der Euler-Punkte $H, S, M, F$ und der Potenzpunkte $1,2, \ldots, 9,1^{*}, 2^{*}, \ldots, 5^{*}$ lassen sich nun weitere Eigenschaften erschließen. So folgt zum Beispiel: Die Dreiecke $M 1^{*} 2^{*}$ und 834 sind zueinander ähnlich mit dem Ähnlichkeitszentrum $S$ (man verwende (2.4) und (2.7) aus Satz 4).

\section{Bemerkungen}

(1) Nach Abschnitt 6 bestehen die Kreistripel der Klasse $K^{* *}$ aus Kreisen $k(P, Q, R)$ durch je drei Punkte der Punktmenge $\left\{A, B, C, S_{A}, S_{B}, S_{C}\right\}$. Wendet man auf $k(A, B, C)$ die zyklischen Vertauschungen (B) aus Abscnitt 4 an, so entsteht kein Kreistripel. Dasselbe gilt für $k\left(S_{A}, S_{B}, S_{C}\right)$. Die Umkreise der Dreiecke $A B C$ und $S_{A} S_{B} S_{C}$ treten daher in der Kreistripelklasse $K^{* *}$ 
nicht auf. $K^{* *}$ enthält genau die fünf verschiedenen Kreistripel der folgenden Tabelle:

\begin{tabular}{c|c} 
Potenzpunkt & Kreistripel \\
\hline $1^{* *}$ & $k\left(A, S_{C}, S_{A}\right) \rightarrow k\left(B, S_{A}, S_{B}\right) \rightarrow k\left(C, S_{B}, S_{C}\right)$ \\
$2^{* *}$ & $k\left(A, S_{A}, S_{B}\right) \rightarrow k\left(B, S_{B}, S_{C}\right) \rightarrow k\left(C, S_{C}, S_{A}\right)$ \\
$3^{* *}$ & $k\left(A, S_{B}, S_{C}\right) \rightarrow k\left(B, S_{C}, S_{A}\right) \rightarrow k\left(C, S_{A}, S_{B}\right)$ \\
$4^{* *}$ & $k\left(A, C, S_{C}\right) \rightarrow k\left(B, A, S_{A}\right) \rightarrow k\left(C, B, S_{B}\right)$ \\
$5^{* *}$ & $k\left(A, B, S_{B}\right) \rightarrow k\left(B, C, S_{C}\right) \rightarrow k\left(C, A, S_{A}\right)$
\end{tabular}

Der Mittelpunkt der Strecke $\overline{1^{* *} 2^{* *}}$ ist der Euler-Punkt $F$. Der Potenzpunkt $3^{* *}$ ist der Euler-Punkt $M$. Die Potenzpunkte $4^{* *}, 5^{* *}$ sind keine EulerPunkte.

(2) Zur Definition weiterer Kreistripel kann man die Punkte $A, B, C, S_{A}, S_{B}, S_{C}$ durch andere Vorgaben ersetzen, z.B. durch $A, B, C$ und die Höhenfußpunkte des Dreiecks $A B C$.

(3) Das Auftreten kollinearer Potenzpunkte in Satz 3 und Satz 4 führt zu der Frage nach der Kollinearität der Potenzpunkte von drei beliebigen Kreistripeln. Die dafür kennzeichnende Bedingung folgt aus der Kollinearitätsbedingung für drei Punkte der euklidischen Ebene unter Verwendung der Formeln (4).

(4) Sei $T$ der Potenzpunkt der drei Ankreise eines Dreiecks $A B C$. Welche Beziehungen bestehen zwischen $T$ und anderen merkwürdigen Punkten des Dreiecks $A B C$ ?

(5) Zu den merkwürdigen Punkten eines Dreiecks $A B C$ zählen auch sein Inkreismittelpunkt $I$, der Inkreismittelpunkt $J$ seines Seitenmittendreiecks $S_{A} S_{B} S_{C}$ und sein Nagel-Punkt $N$. Neben $H, S, M, F$ sind auch $N, S, I, J$ kollineare Punkte (siehe etwa [1], [2]). Welche Potenzpunkte liegen auf ihrer Verbindungsgeraden?

\section{Literatur}

[1] P. Baptist, Nagel-Punkte und Eulersche Geraden, DdM 2 (1982), 114-122.

[2] D. R. Hofstadter, Discovery and Dissection of a geometric Gem, in: Geometry Turned On! Dynamic Software in Learning, Teaching, and Research, (J. R. King, D. Schattschneider, eds.), The Math. Ass. of America, 1997, 3-14.

[3] M. Koecher and A. Krieg, Ebene Geometrie, 2. Aufl., Springer Berlin Heidelberg, 2000. 
[4] K. Kommerell, Vorlesungen über Analytische Geometrie der Ebene, 3. Aufl., Koehler \& Amelang Leipzig, 1953.

[5] H. Martini, Neuere Ergebnisse der Elementargeometrie, in: Geometrie und ihre Anwendungen, (O. Giering and J. Hoschek, eds.), C. Hanser München, 1994, 9-42.

[6] M. Simon, Über die Entwicklung der Elementar-Geometrie im XIX. Jahrhundert, B. G. Teubner Leipzig, 1906.

[7] http://mathworld.wolfram.com.

OSWALD GIERING

D-85591 VATERSTETTEN

JOHANN STRAUSS STRASSE 30

GERMANY

(Received September, 2004) 